\title{
Proton implantation-induced intermixing of InAs/InP quantum dots
}

\author{
S. Barik, ${ }^{a)}$ H. H. Tan, and C. Jagadish \\ Department of Electronic Materials Engineering, Research School of Physical Sciences and Engineering, \\ The Australian National University, Canberra, Australian Capital Territory 0200, Australia
}

(Received 24 January 2006; accepted 3 May 2006; published online 30 May 2006)

\begin{abstract}
Proton implantation-induced intermixing of InAs quantum dots (QDs) capped with InP, GaInAsP, and $\mathrm{InP}$ and $\mathrm{InGaAs}$ layers grown by metal-organic chemical vapor deposition is investigated. The samples are annealed at $750,800,850$, and $900{ }^{\circ} \mathrm{C}$ for $30 \mathrm{~s}$ and thermal stability of the QDs is studied. The optimum annealing temperature is around $800^{\circ} \mathrm{C}$ which gives maximum implantation-induced energy shift. The QDs capped with InP layers show the highest implantation-induced energy shift due to strong group V interdiffusion whereas the QDs grown on and capped with GaInAsP layers show the least implantation-induced energy shift due to weak group V and group III interdiffusion. The QDs capped with InP and InGaAs layers show intermediate implantation-induced energy shift and are less thermally stable compared to the QDs grown on and capped with GaInAsP layers. The QDs capped with InP layers show enhanced photoluminescence (PL) intensity when implanted with lower proton dose (less than 5 $\times 10^{14}$ ions $\left./ \mathrm{cm}^{2}\right)$. On the other hand higher proton dose (more than $1 \times 10^{14}$ ions $\left./ \mathrm{cm}^{2}\right)$ reduces the PL linewidth in all samples. (C) 2006 American Institute of Physics. [DOI: 10.1063/1.2208371]
\end{abstract}

Self-assembled InAs quantum dots (QDs) grown on InP substrates are potential active materials for lasers, semiconductor optical amplifiers for optical fiber telecommunications $(1.3-1.55 \mu \mathrm{m})$, and devices for atmospheric pollution control systems. However, it is still challenging to selectively tune the band gap of the QD structures to monolithically integrate active/passive components of optoelectronic devices for optical communication systems. One of the two main approaches is selective area regrowth technique which involves many processes, e.g., masking, etching, and epitaxy, and it is not cost effective for large scale integration. On the other hand, intermixing is simple which relies on the postgrowth interdiffusion of atoms of QD or quantum well (QW) and barrier layers. Ion implantation-induced intermixing technique has been widely used ${ }^{1-3}$ to modify the potential profile and thereby the emission wavelength of a QD or QW. Furthermore, ion implantation is compatible to planar processing. It provides sufficient lateral resolution so that one can precisely select the QD or QW area to be intermixed. The degree and depth of intermixing can be controlled by varying the implant conditions, e.g., ion dose, ion energy, implantation temperature, and mask profile. Proton implantation-induced intermixing is carried out on InAs QDs grown on and capped with InP, grown on InP and capped with InP and InGaAs layers, and grown on and capped with GaInAsP and the results are presented in this letter. There have been a few reports on postgrowth intermixing in InAs/InP QD structures by rapid thermal annealing and laser irradiation techniques, ${ }^{4}$ and low-energy phosphorous ion implantation induced intermixing in InAs/InP quantum sticks ${ }^{5}$ but there have been no reports of proton implantation induced intermixing in InAs/InP QD structures. Protons are preferred for promoting intermixing since they mainly generate point defects in comparison to other heavier ions. In a recent report, ${ }^{5}$ the quantum stick samples were rapidly thermally annealed at $650{ }^{\circ} \mathrm{C}$.

\footnotetext{
${ }^{\text {a)} E l e c t r o n i c ~ m a i l: ~ s n b 109 @ r s p h y s s e . a n u . e d u . a u ~}$
}

In this letter we report the results of rapid thermal annealing of the QDs up to $900{ }^{\circ} \mathrm{C}$. It has been shown that capping and buffer materials such as InP and GaInAsP change the InAs/InP QDs nucleation, composition, and emission wavelength. ${ }^{6}$ In InGaAs/InP QW system, an InGaAs cap layer increases the thermal stability of the QW compared to an InP cap layer. ${ }^{7}$ In this letter we also present the results of thermal stability and intermixing of InAs/InP QD system with a top InGaAs layer. Our results show that the InGaAs layer does not improve thermal stability of the QDs but it reduces the degree of QD intermixing.

The InAs QDs are grown on (100) semi-insulating (SI) InP substrates on either InP or lattice-matched $\mathrm{Ga}_{0.25} \mathrm{In}_{0.75} \mathrm{As}_{0.54} \mathrm{P}_{0.46}\left(\lambda_{g}=1.28 \mu \mathrm{m}\right)$ buffer using horizontal flow low pressure metal-organic chemical vapor deposition (MOCVD). Trimethylindium (TMI), trimethylgallium (TMG), $\mathrm{PH}_{3}$, and $\mathrm{AsH}_{3}$ are used as precursors, and $\mathrm{H}_{2}$ as the carrier gas. Figure 1 shows schematic representations of the three samples used in this study. Following an oxide desorption step of the substrate at $700{ }^{\circ} \mathrm{C}$ for $10 \mathrm{~min}$ under $\mathrm{PH}_{3}$, the reactor temperature is reduced to $650{ }^{\circ} \mathrm{C}$ and a $200 \mathrm{~nm}$ InP layer is grown. Then either a $500 \mathrm{~nm}$ thick InP or lattice matched GaInAsP layer is grown. To minimize As/P exchange reaction a $0.6 \mathrm{~nm}$ thick GaAs interlayer is grown at the same temperature before the growth of the QD layer at $520^{\circ} \mathrm{C}$. The InAs QDs are grown at the rate of $0.16 \mathrm{~nm} / \mathrm{s}$

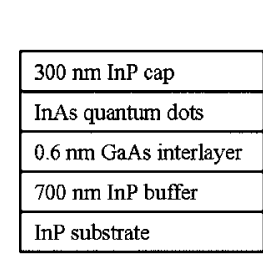

(a)

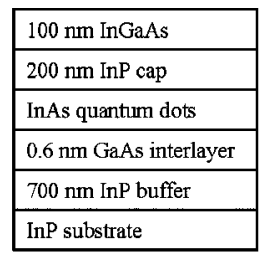

(b)

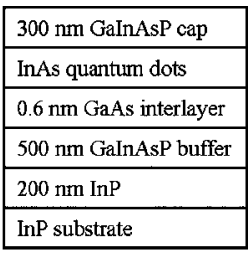

(c)
FIG. 1. Schematic representations of the quantum dot (QD) structures used in this study. The QDs are (a) grown on and capped with InP, (b) grown on InP and capped with InP and InGaAs, and (c) grown on and capped with GaInAsP. 


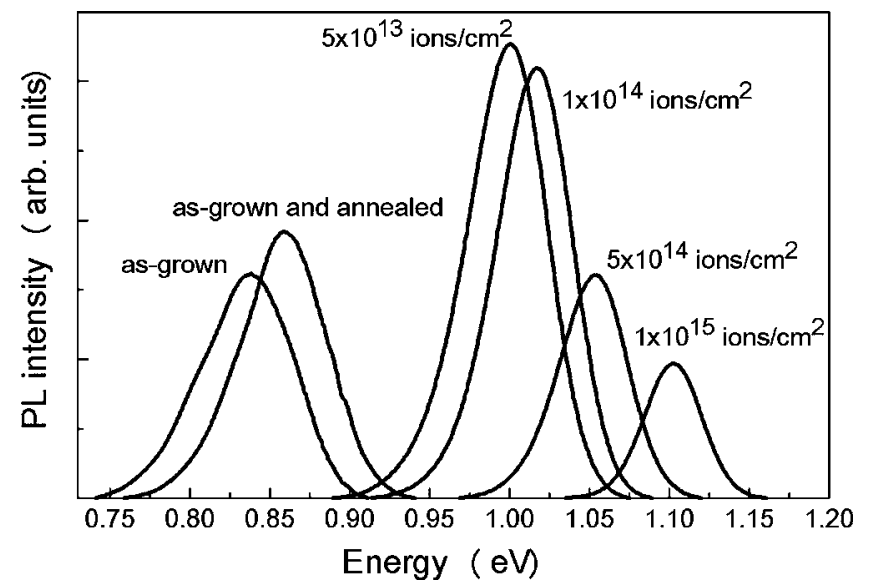

FIG. 2. Photoluminescence spectra measured at $77 \mathrm{~K}$ from the as-grown, as-grown and annealed, and proton-implanted (dose: $5 \times 10^{13}-1$ $\times 10^{15}$ ions $/ \mathrm{cm}^{2}$ ) and annealed quantum dots grown on and capped with InP layers. The rapid thermal annealing is carried out at $800^{\circ} \mathrm{C}$ for $30 \mathrm{~s}$.

using a V/III ratio of 8 . For the InP buffer sample, the growth time of InAs QDs is $6.5 \mathrm{~s}$ and they are immediately capped without any growth interruption with a $300 \mathrm{~nm} \mathrm{InP}$ layer while the temperature is increased to $650{ }^{\circ} \mathrm{C}$. In another sample, instead of a $300 \mathrm{~nm}$ InP layer, a $200 \mathrm{~nm}$ InP layer is grown followed by a $100 \mathrm{~nm}$ lattice-matched InGaAs to check if an InGaAs top capping layer changes thermal stability and intermixing in InAs/InP QD system. For the GaInAsP buffer sample, the growth time of InAs QDs is $5 \mathrm{~s}$ and they are immediately capped without any growth interruption with a $14 \mathrm{~nm}$ GaInAsP layer at $520^{\circ} \mathrm{C}$ and then the growth temperature is ramped up to $650{ }^{\circ} \mathrm{C}$ and the cap layer is terminated with a $286 \mathrm{~nm}$ GaInAsP layer.

Atomic force microscopy and plan-view transmission electron microscopy are carried out to measure the height and size of the QDs. The QDs grown on an InP buffer have a mean height of $8.2 \mathrm{~nm}$, whereas the QDs grown on a GaInAsP buffer have a mean height of $7.8 \mathrm{~nm}$. The mean diameter of the QDs grown on both the buffers is $25 \mathrm{~nm}$.

Proton implantation is performed at room temperature with doses of $5 \times 10^{13}-1 \times 10^{15}$ ions $/ \mathrm{cm}^{2}$. The energy of the ions is $40 \mathrm{keV}$ which places the damage peak over the QD region. To minimize the channeling effect, the implantation is carried out at $7^{\circ}$ off the $\langle 100\rangle$ direction. To remove the damage caused by the implantation and to initiate intermixing, the implanted samples are annealed for $30 \mathrm{~s}$ at 750, 800, 850 , and $900{ }^{\circ} \mathrm{C}$ in argon ambient using a rapid thermal processor. During annealing, the samples are protected from excessive loss of group $\mathrm{V}$ atoms by sandwiching them between two fresh pieces of semi-insulating InP substrates covered with a thick layer similar to the cap layer.

Low temperature $(77 \mathrm{~K})$ photoluminescence (PL) measurements are carried out by exciting with a $532 \mathrm{~nm}$ line of a frequency doubled diode pumped solid state (DPSS) laser. The PL signal is collected by a thermoelectrically cooled InGaAs photodetector with a built-in preamplifier after being dispersed through a $0.5 \mathrm{~m}$ monochromator.

The PL spectra of the as-grown sample and samples annealed at $800{ }^{\circ} \mathrm{C}$ for $30 \mathrm{~s}$ are shown in Fig. 2. The PL recovery in the samples implanted with lower proton dose (less than $5 \times 10^{14}$ ions $/ \mathrm{cm}^{2}$ ) is excellent with even improved PL intensity compared to those of the as-grown, and as-grown and annealed samples. This property of enhanced PL intenDownloaded 25 Jul 2006 to 150.203.178.34. Redistribution subject

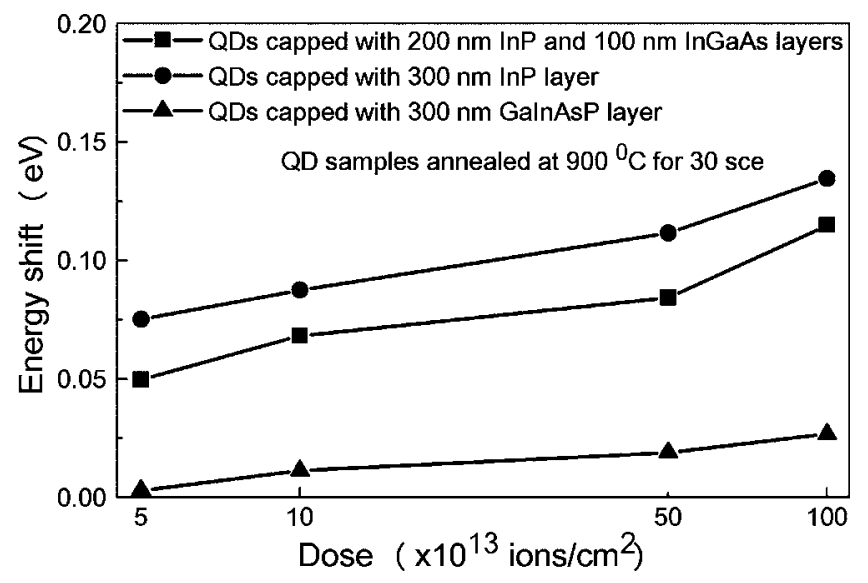

FIG. 3. The implantation-induced energy shifts of the quantum dots capped with InP, InP and InGaAs, and GaInAsP layers and annealed at $900{ }^{\circ} \mathrm{C}$ for $30 \mathrm{~s}$. The implantation dose is varied from $5 \times 10^{13}$ to $1 \times 10^{15}$ ions $/ \mathrm{cm}^{2}$.

sity is observed in all the samples implanted with lower doses for all annealing temperatures from 750 to $900{ }^{\circ} \mathrm{C}$. The implantation-induced intermixing improves the carrier collection efficiency ${ }^{1}$ and possibly residual hydrogen also passivates the nonradiative trapping centers. ${ }^{3}$ Both these effects contribute to the enhanced PL intensity from the samples implanted with lower doses. However, for the samples implanted with higher doses residual defects may still be present after rapid thermal annealing and thereby these samples show reduced PL intensity. Nevertheless, the samples implanted with the highest dose, i.e., 1 $\times 10^{15}$ ions $/ \mathrm{cm}^{2}$, still show a very good PL intensity recovery. As shown in Fig. 2, the as-grown sample annealed at $800{ }^{\circ} \mathrm{C}$ shows $20 \mathrm{meV}$ energy shift with respect to the asgrown sample. The additional energy shift due to implantation increases from 142 to $244 \mathrm{meV}$ as the implantation dose varied from $5 \times 10^{13}$ to $1 \times 10^{15}$ ions $/ \mathrm{cm}^{2}$.

Figure 3 shows the implantation-induced energy shifts of the QDs capped with different layers and annealed at $900{ }^{\circ} \mathrm{C}$ for $30 \mathrm{~s}$ for various implantation doses. The implantationinduced energy shift is defined as the difference between the PL energy peaks of the implanted and unimplanted samples which are annealed at the same temperature. The energy shift increases with increasing implantation dose in all the samples. Similar behavior has been observed in other QD samples annealed at $750-850{ }^{\circ} \mathrm{C}$ for $30 \mathrm{~s}$. The QDs grown on and capped with InP layers show the highest energy shift, whereas the QDs grown on and capped with GaInAsP layers show the least energy shift. In the former case, only group V interdiffusion is responsible for the energy shift. Since there are very high As and $\mathrm{P}$ concentration gradients across the QDs and surrounding layers, the degree of intermixing is higher in this case compared to the QDs grown on and capped with GaInAsP layers. In the latter case, though group III and group V interdiffusions take place, intermixing does not lead to a large energy shift due to the smaller concentration gradient between the dot layer and surrounding layers. The QDs grown on an InP buffer and capped with $200 \mathrm{~nm}$ InP and $100 \mathrm{~nm}$ InGaAs layers show less energy shift compared to the QDs grown on and capped with InP layers though the QDs have InP as the barrier. It has been reported

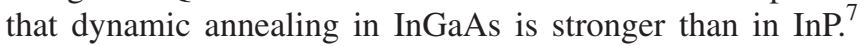
The dynamic annealing is the healing of implant damage which takes place during the implantation process. Since the to AIP license or copyright, see http://apl.aip.org/apl/copyright.jsp 


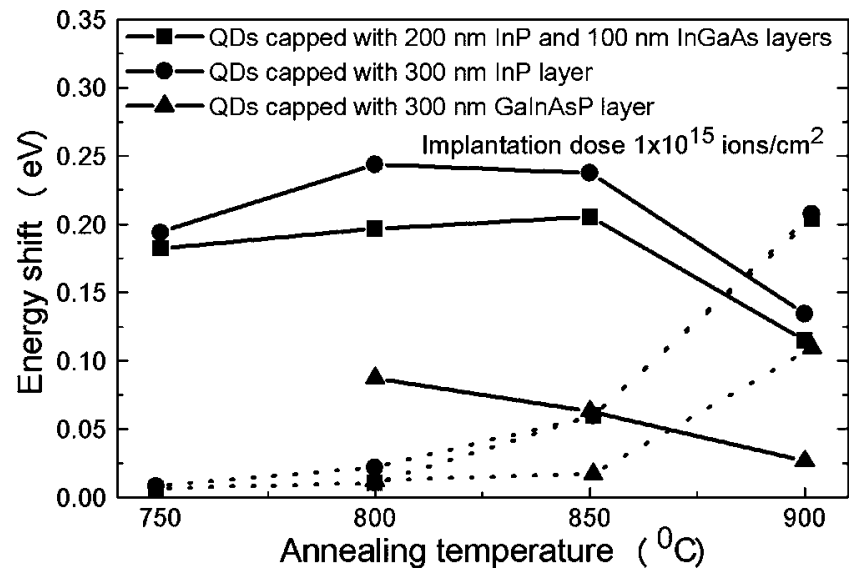

FIG. 4. The solid lines represent the implantation-induced energy shifts of the quantum dots (QDs) capped with InP, InP and InGaAs, and GaInAsP layers. The implantation dose is $1 \times 10^{15}$ ions $/ \mathrm{cm}^{2}$. The dotted lines represent the thermal energy shift of the same but unimplanted samples. The thermal energy shifts of the QDs capped with InP, and InP and InGaAs layers are quite same. The rapid thermal annealing is carried out for $30 \mathrm{~s}$ at different temperatures.

proton implantation creates mainly point defects and the heat supplied to the sample during implantation makes these point defects more mobile, a significant number of vacancies and interstitials may recombine during the implantation process. The effect of the dynamic annealing is higher for the implantation at the elevated temperatures, ${ }^{2}$ but in InGaAs, the dynamic annealing has a significant effect even at room temperature and thereby decreases the concentration of point defects. ${ }^{7}$ Because the degree of intermixing depends on the concentration of point defects present before annealing, the PL energy shift due to intermixing also decreases. Thereby the QDs capped with $300 \mathrm{~nm}$ InP undergo more implantation-induced intermixing and show more energy shift compared to the QDs capped with $200 \mathrm{~nm} \mathrm{InP}$ and $100 \mathrm{~nm}$ InGaAs layers.

The implantation-induced energy shifts of the QDs capped with different layers and annealed at four different temperatures are shown in Fig. 4. Although the overall energy shift increases with increasing annealing temperature, the implanted samples annealed at temperatures around $800{ }^{\circ} \mathrm{C}$ show the highest energy shift. The implanted samples annealed at $750{ }^{\circ} \mathrm{C}$ show intermediate energy shift since annealing at $750{ }^{\circ} \mathrm{C}$ for $30 \mathrm{~s}$ is not sufficient enough to fully induce the implantation-induced intermixing process. However, as shown by the dotted lines in Fig. 4, higher annealing temperature enhances thermal interdiffusion which thereby reduces the differential energy shift, i.e., the energy shift caused by ion implantation. Low temperature annealing (less than $800^{\circ} \mathrm{C}$ ) gives almost no thermal energy shift. Almost no thermal energy shift was also observed in the InAs/InP quantum sticks annealed at $650{ }^{\circ} \mathrm{C}$ but these sticks showed a large energy shift for annealing temperature above $700{ }^{\circ} \mathrm{C} .5$ In our case the QDs grown on and capped with GaInAsP layers are most thermally stable giving less thermal energy shift compared to the energy shift in the QDs grown on and capped with InP layers. Less composition gradient across the QD and GaInAsP barrier layers also plays a role in giving less thermal energy shift. The QDs capped with InP and InGaAs layers show similar thermal energy shift as the QDs capped with InP layer. So an InGaAs top capping layer does not improve thermal stability of the QDs though it improves the thermal stability of QWs. ${ }^{7}$

The QDs grown on and capped with GaInAsP layers require higher temperature annealing $\left(800{ }^{\circ} \mathrm{C}\right.$ and higher) to sufficiently recover the PL intensity. It could be that the same implantation dose creates more (complex) defects in GaInAsP than in InP and/or defect removal in GaInAsP is not as efficient as in InP. The PL intensity recovery of the sample annealed at $750{ }^{\circ} \mathrm{C}$ is too weak to determine the PL peak position and therefore no energy shift is shown in Fig. 4 for this sample. So the optimum annealing temperature could be around $800{ }^{\circ} \mathrm{C}$ considering both the PL intensity recovery and enhanced thermal interdiffusion.

In all the cases, the linewidth of the PL spectra decreases as the proton implantation dose increases above 1 $\times 10^{14}$ ions $/ \mathrm{cm}^{2}$. A similar reduction in the PL linewidth was also observed in proton irradiation-induced intermixing of InGaAs/GaAs QDs (Ref. 2) and low energy phosphorous ion implantation-induced intermixing of the InAs/InP quantum sticks. ${ }^{5}$ A similar effect in laser irradiated and rapid thermal annealed InAs/InP QD samples has also been reported. ${ }^{4}$ Intermixing process particularly induced by higher dose implantation leads to a more uniform QD distribution in terms of shape, size, composition, and strain resulting into reduction in the PL linewidth, since the diffusion process tends to average out all the inhomogeneities.

In summary, we have presented the results of the proton implantation-induced intermixing of InAs/InP QDs. The highest energy shift is observed in the QDs grown on and capped with InP layers and the least energy shift is observed in the QDs grown on and capped with GaInAsP layers. The QDs grown on InP and capped with InP and InGaAs layers show intermediate energy shift. The number of defects formed in different layers can also play a role in the intermixing induced-energy shift. The annealed QDs grown on and capped with InP layers show enhanced PL intensity when implanted with lower dose (less than 5 $\times 10^{14}$ ions $/ \mathrm{cm}^{2}$ ). On the other hand higher dose (more than $1 \times 10^{14}$ ions $/ \mathrm{cm}^{2}$ ) implantation decreases the PL linewidth in all the samples due to homogenization of the QDs as a result of interdiffusion. The implanted samples annealed around $800{ }^{\circ} \mathrm{C}$ show maximum implantation-induced $\mathrm{PL}$ peak energy shift. Though higher temperature annealing reduces the implantation-induced energy shift due to the enhanced thermal interdiffusion, it improves the PL intensity recovery. Our results show that proton implantation can be useful to tune band gap in selected area and thereby to monolithically integrate QD based optoelectronic devices.

The authors gratefully acknowledge financial support from the Australian Research Council.

${ }^{1}$ L. V. Dao, M. Gal, C. Carmody, H. H. Tan, and C. Jagadish, J. Appl. Phys. 88, 5252 (2000).

${ }^{2}$ P. Lever, H. H. Tan, C. Jagadish, P. Reece, and M. Gal, Appl. Phys. Lett. 82, 2053 (2003).

${ }^{3}$ W. Lu, Y. L. Ji, G. B. Chen, N. Y. Tang, X. S. Chen, S. C. Shen, Q. X. Zhao, and M. Willander, Appl. Phys. Lett. 83, 4300 (2003).

${ }^{4}$ C. K. Chia, S. J. Chua, S. Tripathy, and J. R. Dong, Appl. Phys. Lett. 86, 051905 (2005).

${ }^{5}$ B. Salem, V. Aimez, D. Morris, A. Turala, P. Regreny, and M. Gendry, Appl. Phys. Lett. 87, 241115 (2005).

${ }^{6}$ S. Barik, H. H. Tan, and C. Jagadish, Nanotechnology 17, 1867 (2006).

${ }^{7}$ C. Carmody, H. H. Tan, and C. Jagadish, J. Appl. Phys. 93, 4468 (2003). 